\title{
Distribución y densidad de dos especies de holoturoideos en la isla de Cubagua, Venezuela
}

\author{
Alejandro Tagliafico ${ }^{1}$, María Salomé Rangel ${ }^{2} \&$ Néstor Rago $^{3}$ \\ 1. Escuela de Ciencias Aplicadas del Mar, Núcleo de Nueva Esparta, Universidad de Oriente, Boca de Río, Isla de \\ Margarita, Venezuela; atagliafico@hotmail.com \\ 2. Departamento de Ciencias, Núcleo de Nueva Esparta, Universidad de Oriente, Guatamare, Isla de Margarita, \\ Venezuela; salome453@gmail.com \\ 3. Maestría en Ciencias Marinas y Costeras. Universidad Nacional de Costa Rica; nestorrago@yahoo.es
}

$$
\text { Recibido 30-VII-2010. Corregido 10-XI-2010. Aceptado 14-XII-2010. }
$$

\begin{abstract}
Distribution and density of two holothurian species in Cubagua Island, Venezuela. Holothurian populations in the Caribbean have been significantly depleted in some areas, and others are still in danger to satisfy international markets. In Venezuela, this marine resource has been illegally exploited since the early 1990s. To contribute with their management and protection, this work analyzed the distribution, abundance and population structure of two commercial holothurians Isostichopus badionotus and Holothuria mexicana in Cubagua Island shores. A total of 52 stations were studied around the island during the year 2008. Surveys included visits to each station, for which a band transect of $50 \mathrm{~m}^{2}$ with four replicates were carried out. The results show that I. badionotus has a higher distribution on the East coast of the island, and is found over Thalassia testudinum beds or bivalve molluscs aggregations, with an average density of $0.011 \mathrm{ind} / \mathrm{m}^{2}$, C.I. $95 \%=0.005-0.017, \mathrm{n}=122$, and a general abundance estimated in $117 \mathrm{ind} / \mathrm{ha}$. H. mexicana has been practically depleted $\left(0.001 \mathrm{ind} / \mathrm{m}^{2}\right.$ C.I. $95 \%=0.0002-0.0013,7.7 \mathrm{ind} / \mathrm{ha}, \mathrm{n}=8$ ). We recommend the maintenance of the total ban adopted in 1997 to assure the protection of these species, mainly because no previous data supported the start of the fishery, and also, because the current densities are still very low when compared to other areas in Venezuela and the Caribbean. These are animals easy to catch, susceptible to overfishing, and with a very low recovery rate; thus, more control is required from the local authorities. Rev. Biol. Trop. 59 (2): 843-852. Epub 2011 June 01.
\end{abstract}

Keywords: Isostichopus badionotus, Holoturia mexicana, sea cucumber, beche-de-mer.

Las holoturias tienen un papel importante dentro de los ecosistemas marinos, se alimentan del sedimento y aprovechan la microbiota, materia orgánica y nutrientes (Sambrano et al. 1990), lo que produce cambios sobre el fondo marino pues evita la estratificación, enriquece los sedimentos con paquetes fecales que pueden contener hasta el doble de nitrógeno que el sedimento circundante, y procesa la materia orgánica originada por las fanerógamas, indigerible para la mayoría de los individuos marinos (Conde et al. 1995).

Este recurso pesquero, posee gran demanda en los mercados asiáticos, donde es consumido seco, salado, crudo y fermentado (Guzmán \& Guevara 2002). Unas 60 especies son pescadas en más de 40 países (Purcell et al. 2010). Sin embargo, algunas características biológicas como madurez tardía, reproducción denso-dependiente, baja tasa de reclutamiento y la facilidad de sus capturas (Bruckner et al. 2003), implican que este recurso sea especialmente susceptible a la sobre-explotación. Desde hace al menos dos décadas, nuevos casos de sobrepesca son reportados cada año en los océanos de todo el mundo (Lokani 1990, Conand \& Hoffschir 1991, Leslie 1992, Steward 1993, Holland 1994, Powell \& Gibbs 
1995, Lokani 1996, Tsuda 1997, Rasolofonirina \& Conand 1998, Baine \& Poh 1999, Samyn 2000, Conand 2001, Aguilar \& Ramírez 2002, Uthicke et al. 2003, Lindsay \& Simpson 2004, Uthicke \& Conand 2005, Hearn \& Pinillos 2006, Otero-Villanueva \& Ngoc 2007, Hasan 2008, 2009, Al-Rashdi \& Claereboudt 2010). Así, poblaciones enteras del Pacífico han sido agotadas, por lo que empresarios coreanos y japoneses iniciaron la búsqueda de nuevas áreas de pesca en Latinoamérica y el Caribe, desarrollando pesquerías ilegales en áreas no tradicionales, donde los habitantes locales no consumen el producto (Guzmán \& Guevara 2002), y se desconocen las características más básicas de sus poblaciones (Buitrago \& Boada 1996).

En Venezuela, desde inicios de la década de los noventa, existe la presencia de compañías coreanas dedicadas a la extracción de holoturias. Ante el vacío legal en que operaban, a mediados de 1994 se les otorgó un permiso temporal, condicionado a la colaboración con institutos de investigación, para la obtención de datos referentes a la pesquería, abundancia, reproducción y crecimiento de las especies explotadas (Rodríguez-Milliet \& Pauls 1998). De ese convenio, el trabajo de Buitrago \& Boada (1996) reporta la extracción de 16840 ejemplares de Isostichopus badionotus (Selenka 1867), en 459 inmersiones realizadas en un área aproximada de 46ha en la región oriental del país, distribuidas entre Araya, Cariaco, Cubagua, La Isleta, Coche y Macanao. Por su parte, León \& Cabrera (1994) señalaron la extracción de un total de 5.8 toneladas de I. badionotus y Holothuria mexicana, entre dos compañías (12 botes) en cinco meses.

Actualmente, la pesca de holoturias está totalmente prohibida (según Gaceta Oficial $\mathrm{N}^{\circ} 195$ del 19 de julio de 1997). Sin embargo, por observaciones directas y decomisos reportados por la Guardia Costera de Cubagua al instituto encargado de regular la pesca (INSOPESCA), se sabe que estas compañías continúan con la extracción de grandes cantidades, con el uso de compresores de buceo. Laboy-Nieves \& Conde (2006) señalan que existe una necesidad urgente de evaluar el recurso para determinar la sostenibilidad de las pesquerías de holoturias en el país. Por lo anterior es que este trabajo pretende describir la distribución, densidad y estructura de tallas de $I$. badionotus y $H$. mexicana (Ludwing 1875) en los alrededores de la isla de Cubagua, Venezuela, con el objetivo de generar información de línea base que permita realizar comparaciones con los estudios iniciales realizados en el país, y contribuir con la regulación de la extracción del recurso.

\section{MATERIALES Y MÉTODOS}

Área de Estudio: La isla de Cubagua se encuentra ubicada en la región nororiental de Venezuela, sobre la plataforma continental, entre los paralelos $10^{\circ} 47^{\prime}-10^{\circ} 51^{\prime} \mathrm{N}$ y $61^{\circ} 8^{\prime}$ $64^{\circ} 14^{\prime}$ 'W; a una distancia aproximada de $8 \mathrm{~km}$ al sur de la Isla de Margarita (Pta. Piedras) y a unos $20 \mathrm{~km}$ al norte de la Península de Araya. Además, tiene una superficie de $22438 \mathrm{~km}^{2}$ con una longitud aproximada de costa de $25 \mathrm{~km}$ (Cervigón 1997).

Trabajo de campo: La zona costera de la isla de Cubagua fue dividida en 52 celdas imaginarias de $1 \mathrm{~km}^{2}$, hasta una profundidad máxima de $18 \mathrm{~m}$. Durante enero-diciembre 2008, se eligieron al azar, y sin reposición, un mínimo de cuatro y un máximo de cinco celdas por mes, hasta cubrir todos los alrededores de la isla. Cada una de las celdas o estaciones de muestreo fue reconocida al ubicar el punto central, y en campo mediante el uso de un Sistema de Posicionamiento Global (GPS). Dentro de cada celda, una pareja de buzos con equipo autónomo, trazó un total de cuatro réplicas al azar de transectas bandas de $10 \mathrm{~m}$ de largo $\mathrm{x}$ $5 \mathrm{~m}$ de ancho $\left(50 \mathrm{~m}^{2}\right.$ cada una), y totalizó un área de muestreo de $200 \mathrm{~m}^{2}$ por celda de muestreo. Entonces, se realizaron 208 transectos banda, para un total de $10400 \mathrm{~m}^{2}$ de substrato evaluado alrededor de toda la isla de Cubagua. Se contaron cada uno de los ejemplares de $I$. badionotus y $H$. mexicana observados; para evitar extraerlos del agua y ocasionarles algún 
tipo de daño, cada individuo fue medido in situ de la boca al ano, con una cinta métrica flexible (Fuente-Betancourt et al. 2001).

Para examinar la relación entre la densidad de pepinos (ind $/ \mathrm{m}^{2}$ ) y el tipo de fondo, fueron categorizadas, mediante estimaciones visuales, cada una de las 52 celdas en función al tipo de substrato dominante observado en cada transecto. Posteriormente, se comparó dicha abundancia utilizando un análisis de varianza desbalanceado de un factor, con seis niveles de substrato. Los tipos de substrato considerados fueron: arena, ostrales, parches coralinos, parches de octocoral, praderas de fanerógamas (Thalassia testudinum) y algas en descomposición.

Para determinar el tipo de distribución de los organismos en el área de estudio, se estimó en primer lugar el cociente entre la varianza y la media de la densidad de organismos observados en campo, para cada una de las especies. Una vez descartado el patrón de distribución uniforme (cociente entre la varianza y la media de la densidad $>1$ ), se determinaron las frecuencias de aparición de organismos por transectas, y las frecuencias esperadas para una distribución binomial negativa (asociada a distribuciones agregadas), y se usó un estimado preliminar de $\mathrm{k}$ (parámetro que describe la medida de agregación de los organismos) mediante la siguiente fórmula:

$$
\hat{k}=\frac{\bar{x}^{2}}{s^{2}-\bar{x}}
$$

Luego, se calculó k por máxima verosimilitud, resolviendo iterativamente, y tratando de igualar las 2 ecuaciones siguientes:

$$
n * \log _{e} *\left(1+\frac{\bar{x}}{\hat{k}}\right)=\sum\left(\frac{A_{(x)}}{\hat{k}+x}\right)
$$

donde, $\mathrm{A}_{(x)}$ es la suma de las frecuencias observadas superiores a $\mathrm{x}$ para cada $\mathrm{x}, \mathrm{y}$ donde $\mathrm{x}$ corresponde al número de organismos encontrados en cada celda de muestreo (Elliot 1977).

Las zonas con elevadas proporciones de jóvenes fueron identificadas cuando el porcentaje de individuos menores o iguales a $15 \mathrm{~cm}$ de longitud total superaban el $65 \%$ de los ejemplares encontrados por celda (en zonas con más de 2 individuos). La talla utilizada para diferenciar jóvenes de adultos $(15 \mathrm{~cm})$ fue elegida en función del rango de talla observado, y por la talla de madurez sexual señalada por León \& Cabrera (1994) para I. badionotus $(18 \mathrm{~cm})$ y la indicada por Guzmán \& Guevara (2003) de 13 y $20 \mathrm{~cm}$ de Lt para I. badionotus y $H$. mexicana respectivamente.

Finalmente, al utilizar el programa ArcView, fueron realizados los mapas de distribución y densidad de ambas especies, clasificando los rangos de dichos mapas por quartiles.

\section{RESULTADOS}

En el $38 \%$ de las estaciones de muestreo hubo presencia de la especie I. badionotus. Fueron observados un total de 122 ejemplares, con un rango de abundancia entre 0-24 individuos por estación. Se detectó una densidad promedio de $0.011 \mathrm{ind} / \mathrm{m}^{2}$ (C.I. 95\% $=0.005$ 0.017 ), lo que corresponde a unos $117 \mathrm{ind} / \mathrm{ha}$, aproximadamente. La mayor densidad registrada $\left(0.12 \mathrm{ind} / \mathrm{m}^{2}\right)$ se observó en la región suroeste de la isla. Sin embargo, es en la zona oriental donde se encuentra el mayor número de celdas con presencia de I. badionotus. En cambio, en el noreste de la isla no fue observado ningún individuo (Fig. 1).

La distribución geográfica de I. badionotus estuvo estrechamente relacionada con el tipo de substrato predominante en cada una de las zonas (ANOVA, $\mathrm{gl}_{\text {res. }}=46, \mathrm{p}=0.03$ ). La especie muestra un patrón de distribución espacial agregada, con un estimado del parámetro de agregación $\mathrm{k}$ de 0.20 (mientras menor es el valor de $\mathrm{k}$, mayor es el grado de agregación de los organismos en el espacio). La mayor densidad de I. badionotus se encontró en praderas de fanerógamas marinas y en 

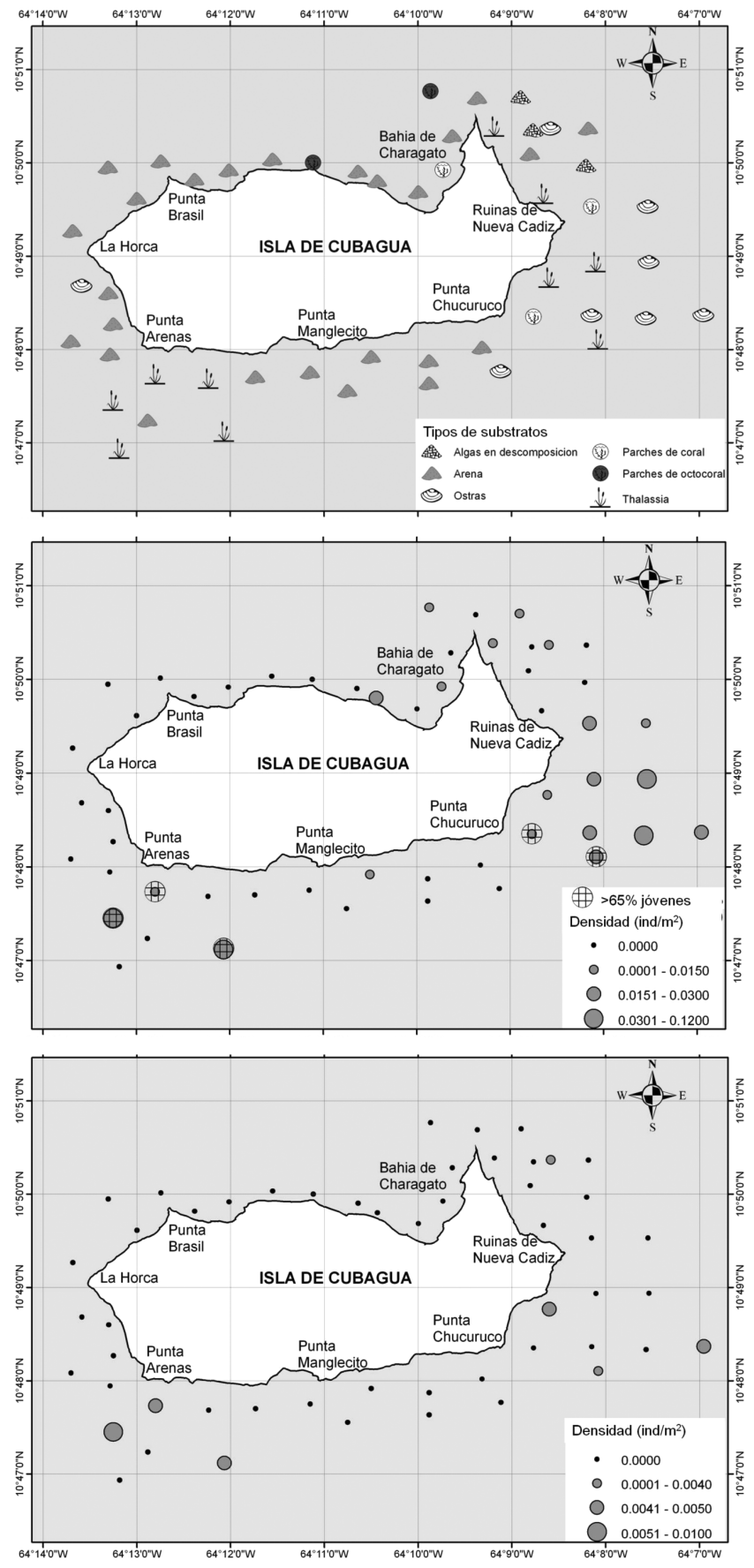

Fig. 1. De arriba hacia abajo: tipos de substrato predominantes en cada estación de muestreo; distribución, densidad (ind $/ \mathrm{m}^{2}$ ) y áreas con elevada proporción de jóvenes de Isostichopus badionotus; distribución y abundancia (ind $/ \mathrm{m}^{2}$ ) de Holothuria mexicana alrededor de la isla de Cubagua.

Fig. 1. From top to bottom: types of predominant substrates observed in each sampling station; distribution, density (ind/ $\mathrm{m} 2$ ) and areas with a high proportion of young Isostichopus badionotus; distribution and abundance (ind $/ \mathrm{m}^{2}$ ) of Holothuria mexicana around the Island of Cubagua. 
ostrales $\left(0.027 \pm 0.04 \mathrm{ind} / \mathrm{m}^{2}\right.$ y $0.024 \pm 0.02 \mathrm{ind} /$ $\mathrm{m}^{2}$, respectivamente); en parches de corales y octocorales se detectó una abundancia intermedia $\left(0.015 \pm 0.01 \mathrm{ind} / \mathrm{m}^{2}\right.$ y $0.008 \pm 0.01 \mathrm{ind} /$ $\mathrm{m}^{2}$, respectivamente); y la menor densidad se observó en comunidades de fondos arenosos y algas descompuestas $\left(0.001 \pm 0.01 \mathrm{ind} / \mathrm{m}^{2} \mathrm{y}\right.$ $0.002 \pm 0.003 \mathrm{ind} / \mathrm{m}^{2}$, respectivamente) (Fig. 2).

I. badionotus presentó un rango de tallas de 7 a $35 \mathrm{~cm}$, con un promedio de $18 \pm 5.3 \mathrm{~cm}$ (Fig. 3). En el $50 \%$ de las estaciones fueron observados adultos y jóvenes juntos; en un $45 \%$ sólo adultos, y un $5 \%$ solo jóvenes. Se identificaron cinco zonas ubicadas al suroeste y sureste de la isla, con más de $65 \%$ de individuos inmaduros (Fig. 1).

Sólo en el $13.5 \%$ de las estaciones de muestreo se detectó la presencia de $H$. mexica$n a$, para un total de ocho individuos en toda el área, con una densidad promedio de $0.001 \mathrm{ind} /$ $\mathrm{m}^{2}$ (C.I. 95\%=0.0002-0.0013) equivalente a unos 7.7ind/ha (Fig. 1). La especie muestra escasa agregación, con coeficiente k de 1.34. No obstante, debido al escaso número de ejemplares analizados, no se realizaron análisis de estructura de talla o de preferencia por algún tipo de substrato. Por lo tanto, su rango de tallas varió entre 8 y $23 \mathrm{~cm}$, con un promedio de $16 \pm 4.7 \mathrm{~cm}$. Se registró un total de seis ejemplares adultos, y dos jóvenes. Todos los individuos fueron observados solitarios, a excepción de una estación donde se encontró un adulto y un juvenil. Además, en todas las estaciones con presencia de $H$. mexicana, también se encontró I. badionotus.

\section{DISCUSIÓN}

Las mayores abundancias y densidades observadas para ambas especies, corresponden a zonas donde el substrato predominante está compuesto por praderas de T. testudinum, lo que coincide con lo reportado en otras áreas del país (Conde et al. 1995). También se encontraron numerosas holoturias en substratos cubiertos por los moluscos bivalvos: Pinctada imbricata Roding 1798 y Arca zebra (Swaison 1833).

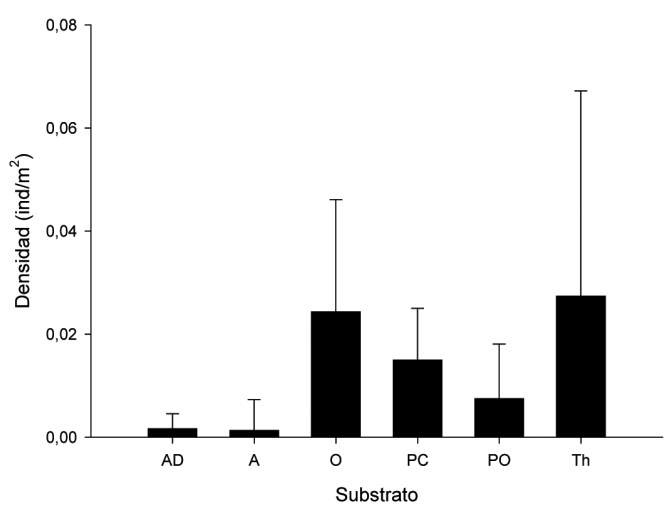

Fig. 2. Densidad promedio de Isostichopus badionotus y el tipo de substrato examinado en las 52 localidades de la isla de Cubagua. AD: algas en descomposición; A: arena; O: ostrales; PC: parches coralinos; PO: parches de octocorales; Th: praderas de fanerógamas marinas.

Fig. 2. Average density of Isostichopus badionotus and type of examined substrate in 52 study sites around Cubagua Island. DS: decomposed seaweeds; S: sand; O: oysters; CP: coralline patches; GP: gorgonian patches; $\mathrm{Sg}$ : seagrass.

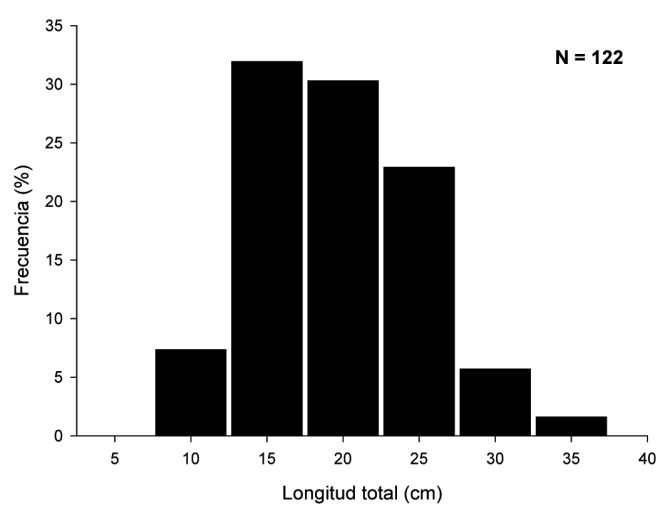

Fig. 3. Estructura de talla de holoturia, Isostichopus badionotus, examinados en la isla de Cubagua en 52 sitios de muestreo.

Fig. 3. Length structure of holoturia, Isostichopus badionotus, examined in Cubagua Island from 52 study sites.

Así pues, I.badionotus habita de forma agregada, hecho que coincide con la reproducción denso-dependiente reportada para este grupo de organismos (Bruckner et al. 2003). Esta especie, muestra una mayor presencia en 
praderas de fanerógamas y ostrales, aunque, fue también observada entre arenales, parches de corales, octocorales y zonas de algas en descomposición, pero en menores densidades. En cambio, H. mexicana solo fue observada de manera dispersa en zonas de fanerógamas $(50 \%)$ y ostrales $(50 \%)$.

En la región este de Cubagua se observó una mayor heterogeneidad de substratos, y más amplia distribución de ambas especies. No obstante, en la zona suroeste destacan dos de las mayores concentraciones observadas para I. badionotus, en la cual se incluyen tres áreas donde más del $65 \%$ de los ejemplares analizados fueron inferiores a $15 \mathrm{~cm}$, y también estuvo presente $H$. mexicana. La presencia de la fanerógama $T$. testudinum, puede explicar estas densidades. En la zona noroeste no fue observado ningún ejemplar, hecho relacionado a la presencia de grandes extensiones de arenales, substrato que demostró tener la menor densidad de holoturias. En el Caribe panameño, ambas especies muestran mayores densidades en áreas con substratos heterogéneos compuestos por pastos marinos y arrecifes coralinos (Guzmán \& Guevara 2002).

Para I. badionotus se estimaron $117 \mathrm{ind} / \mathrm{ha}$, valor superior al calculado con datos provenientes de la pesquería por Buitrago \& Boada (1996) (90ind/ha), e inferior al estimado con los datos de León \& Cabrera en 1994 (entre 530 y 1 100ind./ha).

H. mexicana muestra una disminución en su densidad (7.7ind/ha) en comparación con el rango de 110 a 210ind/ha, observado por León \& Cabrera (1994); la cual coincide en que esta especie es menos abundante que I. badionotus; caso contrario a lo observado en el Caribe panameño (Guzmán \& Guevara 2002). Mientras que, H. mexicana, por alcanzar tallas mayores (Laboy-Nieves \& Conde 2006) puede haber sido extraída en mayores cantidades y esto podría explicar la menor cantidad observada.

Los estudios previos realizados en la zona son escasos, y los que existen fueron desarrollados varios años después de la instalación de empresas coreanas dedicadas a la extracción del recurso en las costas orientales de Venezuela, por lo que la abundancia natural del recurso antes del inicio de la pesquería es desconocida. En otras zonas del país, como el Parque Nacional Morrocoy, se han reportado densidades de $7667 \mathrm{ind} /$ ha (Conde 1997), y el promedio general calculado para el mar Caribe, en el que se incluyen varias localidades ya explotadas por pesquerías, es de 6585 ind/ha (Guzmán \& Guevara 2002), por lo que probablemente las densidades registradas para Cubagua son inferiores a las originales, y corroboran una lenta recuperación del recurso en respuesta a la intensa extracción llevada a cabo antes de que se decretara la veda total de la pesquería.

Las densidades reportadas pueden estar siendo subestimadas, ya que este estudio se llevó a cabo hasta una profundidad máxima de $18 \mathrm{~m}$, pero se sabe que la extracción ilegal se realiza con compresores de aire que poseen mangueras de unos $80 \mathrm{~m}$ de largo, lo que probablemente permite a los pescadores bajar hasta unos $35 \mathrm{~m}$ de profundidad para recolectar las holoturias.

Para I. badionotus, la estructura de tallas fue prácticamente de tipo unimodal con predominio de talla entre los 15 y $20 \mathrm{~cm}$. En general, las poblaciones de holoturias están compuestas de individuos de tallas similares (Sloan \& von Bodungen 1980, Laboy-Nieves \& Conde 2006), entonces los jóvenes son raros de encontrar, o aparecen en poca cantidad (Shiell 2004a), además de presentar reclutamiento bajo y esporádico (Uthicke et al. 2003, Shiell 2004b). Contrariamente, en el lado sur de Cubagua, se observaron zonas con elevadas concentraciones de individuos con tallas inferiores a $\operatorname{los} 15 \mathrm{~cm}(>65 \%)$ y escasa presencia de adultos. El $80 \%$ de estas zonas poseen un substrato predominante de praderas de fanerógamas, las cuales deberían ser consideradas como áreas prioritarias de protección integral para la especie.

Fuente-Betancourt et al. (2001) indican un rango de tallas para $H$. mexicana ubicado entre 17 y $51 \mathrm{~cm}$ para aguas del Caribe mexicano. León \& Cabrera (1994) reportan para la isla de Cubagua tallas máximas cercanas a $30 \mathrm{~cm}$ en las 
dos especies estudiadas. En general, H. mexicana es considerada como una especie de mayor tamaño en comparación con I. badionotus (Laboy-Nieves \& Conde 2006). En el presente trabajo, $H$. mexicana mostró una talla máxima de $21 \mathrm{~cm}$ y $I$. badionotus de $35 \mathrm{~cm}$. Sin embargo, las diferentes metodologías utilizadas para medir las holoturias entre los distintos trabajos, no permiten realizar una mejor comparación.

Implicaciones de manejo: Observaciones directas en el área de estudio permiten inferir que la veda total implantada hace más de diez años (según Gaceta Oficial No 195 del 19 de julio de 1997) no se cumple. En septiembre de 2008 (durante las salidas de muestreo), la Guardia Nacional detuvo una embarcación con buzos que utilizaban un compresor de aire para recolectar holoturias, y donde se decomisaron aproximadamente unos 200 ejemplares, que no fueron devueltos al agua, para ser usados como evidencia del delito. Sumado a esto, la pesca ilícita no solo ocurre en la zona de estudio, puesto que existen numerosas denuncias en los Parques Nacionales: Mochima, Morrocoy, Archipiélago de los Roques y las Aves (Rodríguez-Milliet \& Pauls 1998). Las pesquerías en veda, sin apropiada vigilancia y control, casi nunca cesan por completo, puesto que los elevados precios de exportación del producto para mercados asiáticos, son un fuerte incentivo para que la pesca ilegal continúe, sobre todo en sectores de bajos recursos económicos. Purcell et al. (2010) señalan que los precios de venta por $\mathrm{kg}$ de holoturia seca oscilan entre 300500USD. Por lo general, los elevados precios de venta del producto, en comparación con las bajas multas o la posibilidad de pago de sobornos si son encontrados llevando a cabo pesca ilegal, incentivan a que muchos pescadores tomen el riesgo, por estar conscientes de que las pérdidas son mínimas. Además, es necesario revisar las vías y formatos en que está siendo exportado ilegalmente el producto.

En muchas regiones del mundo, la pesca de pepino de mar aparece repentinamente como una actividad muy lucrativa para los pescadores, lo que trae como consecuencia el rápido declive de las especies objetivos una vez establecida la pesquería. Algunas poblaciones agotadas no se recuperan inclusive después de prohibirse por completo la pesca, y pueden requerir unos 50 años para lograrlo (Bruckner et al. 2003).

$\mathrm{Si}$ asumimos que los alrededores de la isla de Cubagua poseen una superficie aproximada de 45ha, con una abundancia homogénea para todo el área, igual a la observada para I. badionotus (117ind/ha), permitir niveles de explotación similares a los reportados por Buitrago \& Boada (1996) (8hr por bote al día, y capturas de 22ind/hora, ó 176ind/día), podría provocar que un solo bote agote el recurso en tan solo 29 días. Ahora bien, la situación podría ser más grave, ya que la abundancia del recurso no es homogénea, lo que conlleva a que el esfuerzo de pesca se concentre tan sólo en el lado sur y este de la isla. Guzmán \& Guevara (2002) para el Caribe panameño señalan que de permitir niveles de explotación similares a los llevados a cabo en 1997 (25000 ind/día), agotarían las tres especies explotadas en la zona (H. mexicana, I. badionotus y A. multifidus) en tan solo 9 días.

Por lo tanto, se recomienda el monitoreo continuo de las densidades de holoturia en la región oriental del país, además de llevar a cabo estudios de reproducción y reclutamiento de ambas especies. Continuar con la veda total, pero asegurar su cumplimiento, puesto que las densidades de individuos todavía son muy bajas para abrir la pesquería. La Guardia Nacional e INSOPESCA, al realizar decomisos de pesca ilegal, deberían tomar fotografías y devolver inmediatamente al agua los animales, en lugar de conservarlos muertos como evidencia de pesca ilegal.

Las áreas marinas protegidas son efectivas herramientas de manejo que deben ser implementadas junto con otras regulaciones para mantener los niveles estables de las pesquerías (Purcell et al. 2010). La zona protegida más cercana a la isla de Cubagua, es el Parque Nacional Laguna de la Restinga, pero por sus características ecológicas no representa un refugio importante para estas especies. En este 
sentido, Cubagua, ha sido señalada con antiguas riquezas pesqueras actualmente agotadas (Romero et al. 1999), y un alto valor histórico (Cervigón 1997), por lo que existen numerosas justificaciones para protegerla y suficientes atractivos como para que los pescadores residentes puedan obtener beneficios económicos proveniente de actividades relacionadas al turismo. Entonces, se recomienda decretar algunas zonas de veda donde se prohíba por completo la pesca, para poder asegurar la recuperación de estas especies.

En algunos países, ya ha sido planteado utilizar la acuicultura para fines comerciales y de repoblación como solución a la evidente sobreexplotación del recurso (Conand 2001, Toral-Granda et al. 2008, Azari et al. 2010). Inclusive, se han desarrollado co-cultivos exitosos con camarones (Pitt et al. 2004, Pursell et al.2006). Ya que éstos últimos son el principal rubro cultivado del país (Lodeiros \& Freites 2008), habría que evaluar esta posibilidad para ambas especies de holoturia, ya que muestran signos de declive poblacional.

\section{AGRADECIMIENTOS}

Provita y fondo IEA por financiar el proyecto. Alexander Marcano y Alexis Marcano por toda la ayuda brindada en campo. A Jesús Rosas y todo el personal que labora en la estación científica "Fernando Cervigón" de la Universidad de Oriente en la Isla de Cubagua, por todo el apoyo logístico. Régulo López (padre e hijo) por valiosa colaboración. José Silva por su ayuda en la realización de los mapas. Sebastián Rodríguez y Gabriela Pérez por su asesoría. Héctor Guzmán por el envío de artículos sobre el tema. Edlin Guerra por la lectura crítica del manuscrito. Denise Debrot y Richard Parkinson por la revisión del Abstrac. A los revisores anónimos por sus acertadas sugerencias.

\section{RESUMEN}

En algunas zonas del Caribe, poblaciones enteras de holoturias han sido agotadas. En Venezuela, éste recurso ha sido explotado ilegalmente desde inicios de 1990. Por esta razón, se planteó estimar la distribución, abundancia y estructura poblacional de las holoturias comerciales Isostichopus badionotus y Holothuria mexicana en la isla Cubagua. Para ello, durante el año 2008, se realizaron cuatro transectas bandas de $50 \mathrm{~m}^{2}$ cada una en 52 estaciones ubicadas alrededor de la isla. Entre los resultados obtenidos destacan: I. badionotus presenta mayor distribución en el lado este de la isla, sobre fondos de Thalassia testudinum o bancos de moluscos bivalvos; con una densidad promedio de $0.011 \pm 0.022 \mathrm{ind} / \mathrm{m}^{2}(\mathrm{n}=122)$, y una abundancia general estimada en 117ind/ha. Se identificaron cinco posibles zonas de crianza para esta última especie. H. mexicana ha sido prácticamente agotada $\left(0.001 \pm 0.002 \mathrm{ind} / \mathrm{m}^{2}, 7.7 \mathrm{ind} /\right.$ ha, $n=8)$. Se recomienda mantener la veda total implantada en 1997, principalmente por no tener conocimiento de las densidades previas al inicio de la pesquería; por considerar que la densidad actual sigue siendo muy baja en comparación con otras zonas de Venezuela, y por ser animales de fácil captura, rápidamente sobreexplotables y de recuperación lenta.

Palabras clave: Isostichopus badionotus, Holoturia mexicana, pepino de mar, beche-de-mer.

\section{REFERENCIAS}

Aguilar, A. \& G. Ramírez. 2002. Economic reasons, ecological actions and social consequences in the Mexican sea cucumber fishery. SPC Beche-de-mer Infor. Bull. 17: 33-36.

Al-Rashdi, K. \& R. Claereboudt. 2010. Evidence of rapid overfishing of sea cucumbers in the Sultanate of Oman. SPC Beche-de-mer Infor. Bull. 30: 10-13.

Azari, D., B. Giraspy \& I. Walsalam. 2010. Aquaculture potential of the tropical sea cucumbers Holothuria scabra and $H$. lessoni in the Indo-Pacific region. SPC Beche-de-mer Infor. Bull. 30: 29-32.

Baine, M. \& C. Poh. 1999. Sea cucumber fisheries in Malaysia, towards a conservation strategy. SPC Beche-de-mer Infor. Bull. 12: 6-10.

Bruckner, A., K. Johnson \& J. Field. 2003. Conservation strategies for sea cucumber: can CITES Appendix II listing promote sustainable international trade? SPC Beche-de-mer Infor. Bull. 24: 1-10.

Buitrago, J. \& J. Boada. 1996. La pesca de la holoturia Isostichopus badionotus en el oriente de Venezuela. Mem. Soc. Cien. Nat. La Salle 56: 33-40.

Cervigón, F. 1997. Cubagua 500 años. Fundación Museo del Mar, Boca de Río, Nueva Esparta, Venezuela. 
Conand, C. \& C. Hoffschir. 1991. Recent trends in sea cucumbers exploitation in New Caledonia. SPC Beche-de-mer Infor. Bull. 3: 5-7.

Conand, C. 2001. Overview over the last decade of sea cucumber fisheries: what possibilities for a durable management?, p. 339-344. In M. Barker (ed.). Echinoderms 2000: Proceedings of the $10^{\text {th }}$ International Conference. A. A. Balkema, Rotterdam, Holanda.

Conde, J.E. 1997. Après les bébés phoques, les concombres de mer. La Recherche 304: 61-64.

Conde, J.E., A. Sambrano \& H. Díaz. 1995. Sedimertivorous activity, density and spatial distribution of the deposit-feeding holothurians Holothuria mexicana Ludwing and Isostichopus badionotus Selenka. Manuscrito, Caracas, Distrito Federal, Venezuela.

Elliot, J.M. 1977. Some methods for the statistical analysis of samples of benthic invertebrates. Freshwater Biological Association, Ambleside, Inglaterra.

Fuente-Betancourt de la G.M., A. Jesús-Navarrete, E. SosaCordero \& M.D. Herrero-Perezrul. 2001. Assessment of the sea cucumber (Echinodermata; Holothuroidea) as potencial fishery resource in 274 Banco Chinchorro, Quintana Roo, Mexico. Bull. Mar. Sci. 68: 59-67.

Guzmán, H. \& C. Guevara. 2002. Population structure, distribution and abundance of three commercial species of Sea Cucumber (Echinodermata) in Panama. Caribb. J. Sci. 38: 230-238.

Guzmán, H. \& C. Guevara. 2003. Reproductive cycle of two commercial species of sea cucumber (Echinodermata: Holothuroidea) from Caribbean Panama. Mar. Biol. 142: 271-279.

Hasan, M. 2008. Fisheries status and management plan for Saudi Arabian sea cucumbers. SPC Beche-de-mer Infor. Bull. 28: 14-21.

Hasan, M. 2009. Stock assessment of holothuroid populations in the Red Sea waters of Saudi Arabia. SPC Beche-de-mer Infor. Bull. 29: 31-37.

Hearn, A. \& F. Pinillos. 2006. Baseline information on the warty sea cucumber Stichopus horrens in Santa Cruz, Galápagos, prior to the commencement of an illegal fishery. SPC Beche-de-mer Infor. Bull. 24: 3-10.

Holland, A. 1994. The beche-de-mer industry in the Solomon Islands: recent trends and suggestions for management. SPC Beche-de-mer Infor. Bull. 6: 2-9.

Laboy-Nieves, E. \& J.E. Conde. 2006. A new approach for measuring Holothuria mexicana and Isostichopus badionotus for stock assessments. SPC Beche-de-mer Infor. Bull. 24: 39-44.

León, L. \& T. Cabrera. 1994. Informe de avance sobre la pesquería del pepino de mar en las costas del nororiente de Venezuela. Informe Técnico, Escuela de Ciencias Aplicadas del Mar, Universidad de Oriente, Boca de Río, Nueva Esparta, Venezuela.

Leslie, J. 1992. Review of the beche-de-mer (sea cucumber) fishery in the Maldives, Programme Officer, Bay of Bengal Programme (April 1992). Bay of Bengal Programme, Madras, India.

Lindsay, S. \& A. Simpson. 2004. Resource evaluation of current populations of two commercially harvested sea cucumber species (Actinopyga mauritiana and Stichopus chloronotus), and recommendations for management for Kosrae State, Federated States of Micronesia. SPC Beche-de-mer Infor. Bull. 19: 31-33.

Lodeiros, C. \& L. Freites. 2008. Estado actual y perspectivas del cultivo de moluscos bivalvos en Venezuela, p.135-150. In A. Lovatelli, A. Farias \& I. Uriarte (eds.). Estado actual del cultivo y manejo de molus$\cos$ bivalvos y su proyeccion futura: factores que afectan su sustentabilidad en América Latina. FAO Actas de Pesca y Acuicultura. Roma, Italia.

Lokani, P. 1990. Beche-de-mer research and development in Papua New Guinea. SPC Beche-de-mer Infor. Bull. 2: 8-11.

Lokani, P. 1996. Illegal fishing for sea-cucumber (bechede-mer) by Papua New Guinea artisanal fishermen in the Torres Strait protected zone. SPC Beche-de-mer Infor. Bull. 8: 2-6.

Otero-Villanueva, M. \& V. Ngoc. 2007. Sea cucumber fisheries around Phu Quoc Archipelago: A cross-border issue between South Vietnam and Cambodia. SPC Beche-de-mer Infor. Bull. 25: 32-36.

Pitt, R., N. Duy, T. Duy \& H. Long. 2004. Sandfish (Holothuria scabra) with shrimp (Penaeus monodon) co-culture tank trials. Beche-de-mer Infor. Bull. 20: $12-22$.

Powell, J. \& J. Gibbs. 1995. A report from Galapagos. Tree 10: $351-354$.

Pursell, S., J. Patrois \& N. Fraisse. 2006. Experimental evaluation of co-culture of juvenile sea cucumbers, Holothuria scabra (Jaeger), with juvenile blue shrimp, Litopenaeus stylirostris (Stimpson). Aquac. Res. 515-522.

Purcell, S., A. Lovatelli, M. Vasconcellos \& Y. Ye. 2010. Managing sea cucumber fisheries with an 
ecosystem approach. FAO Fish. Aquac. Tech. Paper 520, Roma, Italia.

Rasolofonirina, R. \& C. Conand. 1998. Sea cucumber exploitation in the Toliara region of south-west Madagascar. Beche-de-mer Infor. Bull. 10: 10-13.

Rodríguez-Milliet, E. \& S.M. Pauls. 1998. Sea cucumbers fisheries in Venezuela, p. 513-516. In R. Mooi \& M. Telford (eds.). Echinoderms. Balkema, San Francisco, Estados Unidos.

Romero, A., S. Chilbert \& M. Eisenhart. 1999. Cubagua's Pearl-Oyster beds: the first depletion of a natural resource caused by Europeans in the American Continent. J. Polit. Ecol. 6: 57-78.

Sambrano, A., H. Díaz \& J.E. Conde. 1990. Caracterización de la ingesta en Isostichopus badionotus (Selenka) y Holothuria mexicana (Ludwing). (Echinodermata: Holothuroidea). Caribb. J. Sci. 26: 45-51.

Samyn, Y. 2000. Conservation of aspidochirotid holothurians in the littoral waters of Kenya. SPC Beche-demer Infor. Bull. 13: 12-17.

Shiell, G. 2004a. Field observation of juvenile sea cucumber. SPC Beche-de-mer Infor. Bull. 20: 6-11.

Shiell, G. 2004b. Questionnarie on the field observation of juvenile sea cucumber. SPC Beche-de-mer Infor. Bull. 19: 41.
Sloan, N.A. \& B. von Bodungen. 1980. Distribution and feeding of the sea cucumber Isostichopus badionotus in relation to shelter and sediment criteria of the Bermuda platform. Mar. Ecol. Prog. Ser. 2: 257-264.

Stewart, B. 1993. Evidence for a marked decline of bechede-mer populations in the Suva and Beqa areas of Fiji, and apreliminary description of a method of identifying beche-de-mer individuals based on characteristicbody wrinkles. University of the SouthPacific Marine Studies Technical Report No.1/93. University of Otago, Nueva Zelandia.

Toral-Granda, V., A. Lovatelli \& M. Vasconcellos. 2008. Sea cucumbers. A global review on fishery and trade. Beche-de-mer Infor. Bull. 27: 4-6.

Tsuda, R. 1997. Survey of commercially-valued sea cucumbers in the Saipan Lagoon, CNMI Beche-demer Infor. Bull. 9: 15-17.

Uthicke, S., D. Welch \& J. Benzie. 2003. Slow growth and lack of recovery in overfished holothurians on the Great Barrier Reef: evidence from DNA fingerprints and repeated large-scale surveys. Conserv. Biol. 18: 1395-1404.

Uthicke, S. \& C. Conand. 2005. Local examples of beche-de-mer overfishing: An initial summary and request for information. SPC Beche-de-mer Infor. Bull. 21: 9-14. 